\title{
Photochemistry Meets Porous Organic Cages
}

\author{
Hsin-Hua Huang ${ }^{\S}$ and Tomáš Šolomek* \\ §SCS-Metrohm Award for best oral presentation in Organic Chemistry
}

\begin{abstract}
Chemistry of porous organic cages has developed in the past decade as an alternative to the wellknown nanoporous materials based on extended networks, such as metal organic frameworks (MOFs) or covalent organic frameworks (COFs). Unlike these extended polymeric materials, the molecular nature of organic cages offers important advantages, such as solubility of the material in common organic solvents. However, a simultaneous combination of porosity and additional optoelectronic properties, common in MOFs and COFs, is still quite rare. Therefore, porous organic cages are relatively underdeveloped when compared to MOFs and COFs. Here, we highlight the rich possibilities the porous organic cages offer and discuss the recent development where interesting photophysical properties augment the porosity, including our own work.
\end{abstract}

Keywords: Catalysis · Dynamic covalent chemistry · Photochemistry · Porosity · Porous organic cages

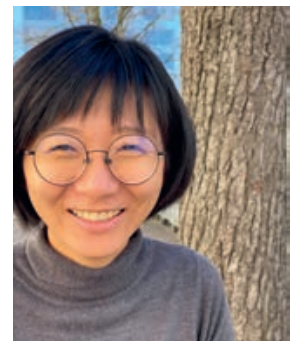

Hsin-Hua Huang was born and raised in Taiwan. She received her master's degree in organic chemistry at National Taiwan University (NTU). During her master studies, she worked on the synthesis of polymeric ladderphanes with ferrocene moiety as linkers. In 2018, she joined the Solomek group as a $\mathrm{PhD}$ student. Her research interest focuses mainly on the rational design and synthesis of chiral and redox-active covalent organic cages and their photophysical properties.

\section{Introduction}

Connecting the nanoscale voids into a network of pores in a material leads to material nanoporosity, which is typically supported by a regular inorganic or organic framework. A large number of such pores endows nanoporous materials with large surface areas that are available for adsorption or chemical reactions that are crucial in a number of industrial applications. Chemists developed ingenious methods to construct porous frameworks using reticular chemistry, ${ }^{[1]}$ where molecular building blocks, known as struts, are linked via nodes leading to extended, polymeric structures. The chemical nature of the struts and nodes can be tuned by synthesis, which opened immense possibilities and led to the blossoming of chemistry of metal-organic frameworks ${ }^{[2]}$ (MOFs) and covalent organic frameworks ${ }^{[3]}$ (COFs) to highlight the bestknown examples. The porous nature of MOFs and COFs can be complemented by redox- or photochemical properties that permit new applications of such materials that go beyond just porosity. For example, the optoelectronic properties of individual building blocks can be utilized to create conductive thin films ${ }^{[4]}$ that could be used in applications including photovoltaics, sensors, and electronic materials. However, both MOFs and COFs represent extended polymeric structures with limited solubility, which hampers processing these materials at low cost into large-area thin films or membranes.
The challenges associated with limited solubility could be circumvented if the regular structure of the nanoporous material was supported by a framework consisting of individual molecules coupled in the solid state only via weak non-bonding interactions. The ideal molecules for such a task are organic cages that possess a molecular void and windows that allow for an exchange of material between the cage cavity and its surroundings. ${ }^{[5]}$ Connecting the voids via these cage windows creates an intrinsic network of channels leading to materials called porous organic cages (POCs). The porosity of POCs, moreover, can be augmented by extrinsic voids created via the packing of cage molecules in the solid-state. A genuine interest in POCs was sparked roughly a decade ago and their synthesis developed rapidly due to their attractive features that combine the properties typical for organic compounds and those inherent to materials based on extended frameworks. Despite our ability to synthesize molecules with very complex architectures, embedding a multivariate functionality into POCs is still in its infancy. Here, we briefly highlight the general advantages of POCs using imine cages as an example and discuss the recent development of POCs that incorporate porphyrins and rylene diimides as chromophores. The photoactive POCs could, in principle, be incorporated into processable nanoporous materials that integrate porosity and photochemistry, a combination useful in applications such as photocatalysis. We hope that this perspective will represent an incentive for such development involving chemists in Switzerland.

\section{Synthesis, Structure, and Porosity of Organic Cages}

In 2009, Cooper and co-workers used dynamic covalent chemistry[6] (DCC, Fig. 1) to synthesize the first tetrahedral POCs 1. ${ }^{[7]}$ DCC relies on reversible covalent bond formation that involves rapid error correction to create the thermodynamically most stable product. This approach circumvents the challenges of long multi-step synthesis that relies on kinetically controlled reactions, which often lead to side-products in each synthetic step and to low overall yields. Suitable choice of reaction partners in DCC, 
in particular their geometry, can then provide complex structures in good yields and purity in a single synthetic step. For example, POCs 1 were obtained by reacting tritopic benzene1,3,5-tricarbaldehyde and ditopic amines in a [4+6]imine condensation process (Fig. 1). After the successful synthesis, the obtained crystalline material contains solvent molecules in its pores. Therefore, the solvent molecules must be first removed to vacate them. Desolvation of $\mathbf{1}$ provided porous samples with Brunauer-Emmett-Teller (BET) surface areas $\left(S A_{\mathrm{BET}}\right)$ of up to $624 \mathrm{~m}^{2}$ per $1 \mathrm{~g}$ of the material. The steric bulk in the used amines affected the pore structure and the packing in the crystal. For instance, cage 1a made from the small 1,2-ethylenediamine and crystallized from ethyl acetate displayed window-to-arene stacks blocking the intercage window-window connectivity, which made 1a a non-porous material, although the cage itself has an intrinsic void. Cage $\mathbf{1 b}$ with an extra methyl group in the amine linker packs less efficiently, reducing the crystallographic density. As a result, one-dimensional pores exist between individual cages providing the extrinsic porosity of the material. Yet, the inner voids remain isolated. Unlike in $\mathbf{1 a}$ and $\mathbf{1 b}$ made from sterically unhindered amines, $1 \mathrm{c}$ is formed from bulky trans-1,2cyclohexanediamine that forces 1c to pack in a window-to-window manner creating a diamondoid three-dimensional system of channels (Fig. 1). However, all cages 1 were able to adsorb gases such as $\mathrm{N}_{2}, \mathrm{H}_{2}, \mathrm{CH}_{4}$, or $\mathrm{CO}_{2}$ with different selectivity after desolvation, and exhibited type I sorption isotherms, although 1a showed diminished uptake of $\mathrm{N}_{2}$ at $77 \mathrm{~K}\left(S A_{\mathrm{BET}}=24 \mathrm{~m}^{2} \mathrm{~g}^{-1}\right)$. This suggests that the internal voids can be accessed by the guest molecules at higher temperature even in the absence of a permanent pore structure. In addition, gas uptake selectivity promises that POCs could be used to separate different gases. POC 1a could be recrystallized from a mixture of dichloromethane and $o$-xylene to obtain a permanently porous polymorph that showed $S A_{\mathrm{BET}}=550 \mathrm{~m}^{2} \mathrm{~g}^{-1}$, which is comparable to those determined for 1b and 1c (Fig. 1).

\section{Synthesis of imine POCs 1 via DCC}
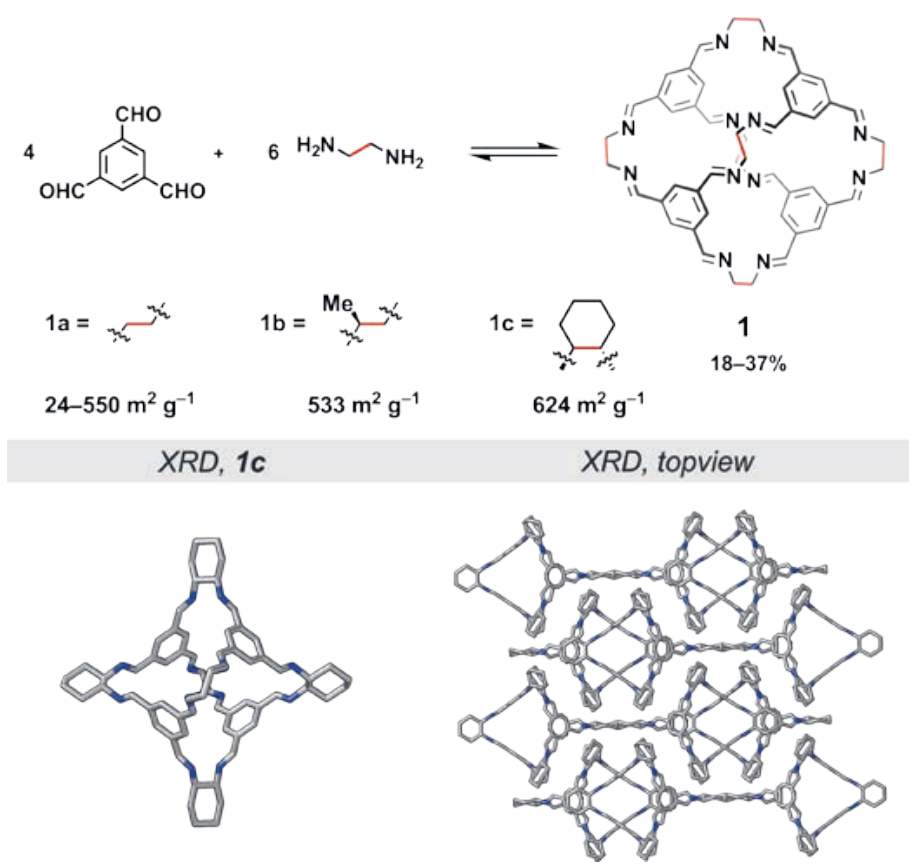

Fig. 1. Structure and synthesis (top) of imine POCs 1 via dynamic covalent chemistry and the single crystal X-ray (XRD) structure of 1c showing the individual cage structure (bottom, left) and its window-to-window packing (bottom, right). Brunauer-Emmett-Teller surface area (in $\mathrm{m}^{2} \mathrm{~g}^{-1}$ ) measured for adsorption of $\mathrm{N}_{2}$ at $77 \mathrm{~K}$ is given. Hydrogen atoms in the crystal structures are omitted for clarity.
Besides adsorption of gases, POCs $\mathbf{1}$ can be used to separate aromatic feedstock molecules that are difficult and costly to separate by distillation due to similar boiling points. For example, triangular mesitylene has a similar molecular volume as its constitutional isomer, linear 4-ethyltoluene. However, the shape and not the size of the aromatic guest molecule together with the cage flexibility are decisive factors controlling their separation. ${ }^{[8]}$ Low flexibility of cage 1c limits the rate of the guest exchange between the cage inner void and its surrounding. For instance, when 1c with physisorbed $\mathrm{CO}_{2}$ was dissolved in mesitylene, $\mathrm{CO}_{2}$ in the cage was not replaced by the solvent molecules unlike in 4-ethyltoluene, where the $\mathrm{CO}_{2}$ was released immediately. Similarly, dissolving a crystal of $\mathbf{1 c}$ crystallized with mesitylene in the cavity of $1 \mathrm{c}$ in $\mathrm{CDCl}_{3}$ allowed to observe a slow release $\left(\tau_{1 / 2}=2.5\right.$ days) of mesitylene from the void in 1c by NMR spectroscopy. 4-Ethyltoluene, however, escaped in an instant upon dissolution. The observed kinetic differences translate into a different diffusibility through crystalline 1c for mesitylene and 4-ethyltoluene. These principles also apply in gas or liquid chromatographic methods where POCs were used as a stationary phase. ${ }^{[9]}$ For example, chiral 1c was utilized to separate krypton, xenon, and radon at concentrations of only a few parts per million from air, ${ }^{\text {[a] }}$ or to separate racemates by gas chromatography. ${ }^{[9 b-d]}$

This seminal series of works highlights the rich possibilities of POCs, such as: a) synthesis using DCC, b) the processability of POCs as organic molecules due to their solubility, the possibility to c) obtain single crystalline material and to d) alter the porosity either by crystal structure engineering or by accessing different polymorphs, and the ability to e) adsorb various gases with different selectivity and to f) efficiently separate aromatic feedstock molecules.

\section{Beyond the Porosity of Porous Organic Cages}

The sufficient condition for the exciting applications that we very briefly discussed above was the presence of a permanent or a dynamic channel in the material to allow for the mass transport. However, applications such as sensing or catalysis go beyond porosity. In MOFs or COFs, porosity helps chemical species to access specific sites where sensing or catalysis take place.[10] However, an extra functionality in POCs is required to enable these processes. POCs that are both porous and possess useful optoelectronic properties are rare. Below, we discuss the few examples of POCs synthesized by DCC that incorporate useful chromophores. In some cases, integration of these chromophores into POCs has already delivered the first examples of POC heterogeneous photocatalysts.

\section{Porphyrin Chromophores}

One of the best-known chromophores with excellent lightharvesting properties is porphyrin.[11] To explore the effect of multiple porphyrin units in an organic cage, Kim and co-workers synthesized cages 2 (Fig. 2) called, according to their shape, porphyrin boxes. The structure of a porphyrin box relies on the fourfold rotational symmetry of the porphyrin unit. Therefore, tetratopic porphyrin tetracarbaldehyde and a tritopic amine provided the large porphyrin boxes in a $[6+8]$ dynamic imine condensation process (Fig. 2). The diameter of the internal cavity in the larger 2a is nearly $2 \mathrm{~nm}$ and the cage has twelve accessible windows. ${ }^{[12]}$ The structures of both porphyrin boxes $\mathbf{2}$ did not collapse upon desolvation. Gas sorption isotherms showed high surface areas $S A_{\mathrm{BET}}=1370 \mathrm{~m}^{2} \mathrm{~g}^{-1}$ and $S A_{\mathrm{BET}}=935 \mathrm{~m}^{2} \mathrm{~g}^{-1}$ for the larger $\mathbf{2 a}$ and smaller $\mathbf{2} \mathbf{b}$, respectively. While the larger $\mathbf{2 a}$ exhibited a moderate $\mathrm{CO}_{2}$ uptake and selectivity over $\mathrm{N}_{2}$, the smaller $\mathbf{2 b}$ showed a superb performance with $\mathrm{CO}_{2} / \mathrm{N}_{2}$ uptake selectivity $>200$. This is in accord with previous literature reports ${ }^{[13]}$ that smaller channels and the presence of amino substituents enhance the sorption of $\mathrm{CO}_{2}$ because it has a smaller kinetic diameter than $\mathrm{N}_{2}, \mathrm{CO}$, or 
Fig. 2. (top) Single crystal X-ray structures of porous organic cages with porphyrin units and (bottom) photocatalytic reactions with porphyrin POCs as heterogeneous photocatalysts. The aldehydes and amines used in the synthesis of porphyrin POCs are shown. aPorphyrins are different porphyrin derivatives - cages $2(5,10,15,20$-tetra4-formylphenylporphyrin), cage 3 (5,10,15,20-tetra-4-aminophenylporphyrin), cage 4 (5,15-di-4'-formylbiphenylporphyrin). Hydrogen atoms and alkyl chains (Rs) in the crystal structures are omitted for clarity.

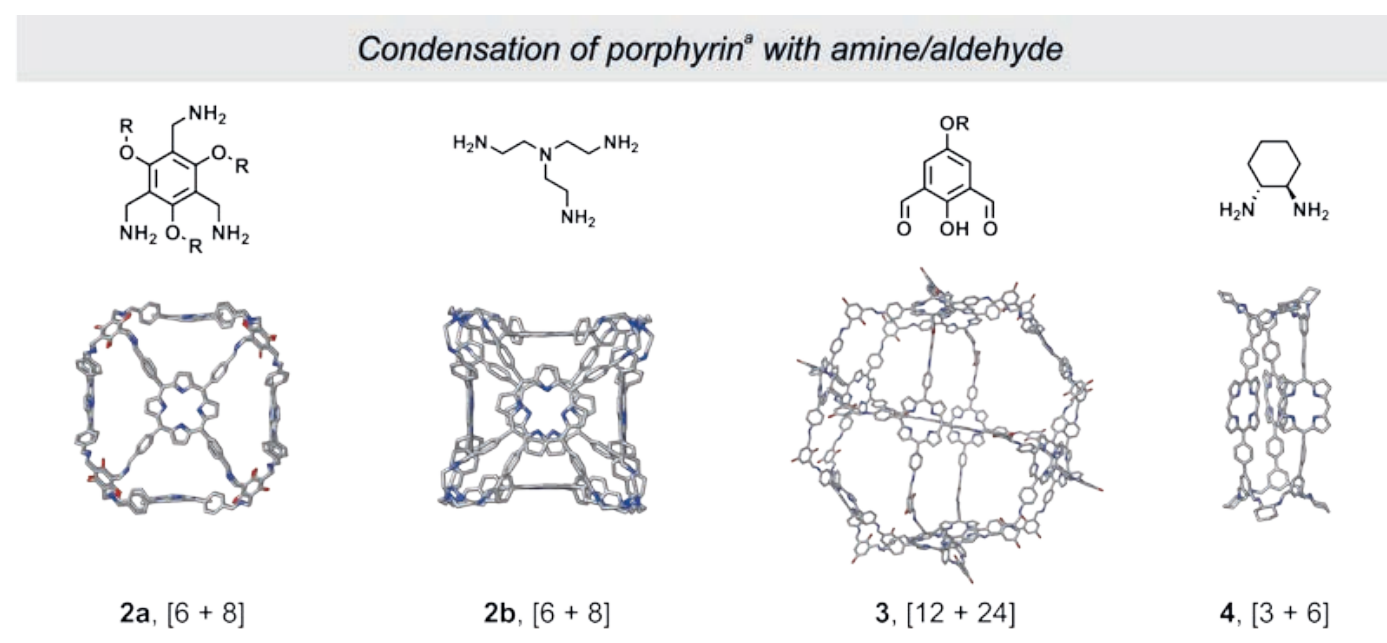

Photocatalytic reactions with porphyrin cages

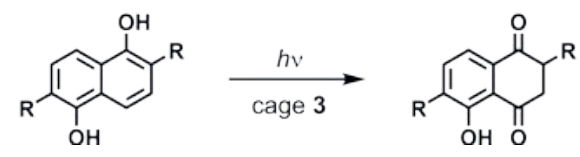

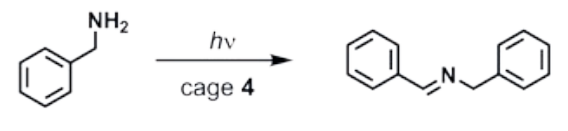

$\mathrm{CH}_{4}$. These results clearly demonstrate that porphyrin boxes are highly porous and could be appealing for applications to separate $\mathrm{CO}_{2}$ from industrial sources such as flue gas.

Kim's group envisaged that charge-transfer interactions between porphyrin and a suitable electron acceptor, such as fullerene, could lead to a hierarchical porphyrin-fullerene superstructure. Indeed, co-crystallization of fullerenes with a porphyrin box created supramolecular fullerene tetramers concocted with the porphyrin boxes, assemblies that are not possible to achieve with a monomeric porphyrin. ${ }^{[14]}$ Due to the unique three-dimensional spatial arrangement, long-lived charge-separated (CS) states were formed by irradiation of the assembly and led to a high measured photocurrent. Formation of long-lived CS states in such donor-acceptor architectures could be utilized in photoredox catalysis or in artificial photosynthesis. Recently, Kim and co-workers reported ${ }^{[15]}$ a one-pot synthesis of a $[12+24]$ porphyrin organic cage 3 (Fig. 2), in which twelve porphyrin units and twenty-four rigid 5-alkoxy-2-hydroxyisophthalaldehyde linkers assemble into one of the largest purely organic cages synthesized so far. The size of $\mathbf{3}$ is $\sim 5.3 \mathrm{~nm}$ and the inner cavity diameter $\sim 4.3 \mathrm{~nm}$. Photoredox activity of POC 3 was tested. It showed much faster photocatalytic oxidation of dihydroxynaphthalene (DHN, Fig. 2) derivatives than a monomeric reference compound or a smaller porphyrin box 2 . Compound 3 displayed even better performance when the size of the DHN derivatives increased. This observation can be ascribed to the larger pore size in $\mathbf{3}$ that allows DHNs to diffuse in the pores, while the larger derivatives get blocked progressively with their size in porphyrin POCs with narrower channels.

Another tubular $[3+6]$ metal-free porphyrin organic cage 4 (Fig. 2) was recently investigated by Jiang and co-workers. ${ }^{[16]}$ It shows a type $\mathrm{I} \mathrm{N}_{2}$ sorption isotherm with $S A_{\mathrm{BET}}=112 \mathrm{~m}^{2} \mathrm{~g}^{-1}$. The authors attributed the lower fluorescence quantum yield and shorter excited state lifetime of the cage when compared to the monomeric reference to an increased rate of intersystem crossing. However, the triplet quantum yields could not be determined. Nevertheless, the triplet lifetime could be obtained by transient absorption spectroscopy and the data showed that the triplet state lifetime in $\mathbf{4}$ is longer $(102 \mu \mathrm{s})$ relative to the monomer porphyrin $(54 \mu \mathrm{s})$. Consequently, the cage exhibited a high formation of reactive oxygen species (ROS) when compared to a monomeric porphyrin. It was, therefore, expected that ROS produced by the cage may play a key role in the photooxidation reactions. Indeed, complete photooxidation of benzylamine (>99\%) into $N$-benzylidenebenzylamine (Fig. 2) with the porphyrin cage as a heterogeneous catalyst was observed within an hour of irradiation. Longer irradiation times were necessary for other tested monomeric porphyrin photocatalysts. Control experiments revealed that ${ }^{1} \mathrm{O}_{2}$ and not $\mathrm{O}_{2}{ }^{-}$was responsible for the observed photooxidation. The photooxidation performance was retained after five cycles, which demonstrates that the POC photocatalyst can be recycled. Despite only a few recent reports in the literature, results of porphyrin POCs as photocatalysts are encouraging and suggest that they exhibit a great potential as supramolecular heterogeneous electro- or photocatalysts.

\section{Rylene Diimide Chromophores}

Instead of electron-rich porphyrins, we turned our attention to electron-deficient rylene diimides. Rylene diimides are well-known as (photo)chemically stable chromophores and as great electron acceptors. ${ }^{[17]}$ Properties of rylene diimides, such as strong absorption/emission or favorable redox potentials in naphthalene diimide (NDI) or perylenediimide (PDI), can be easily modified by synthesis, which enabled these compounds to find a broad range of applications. ${ }^{[17]}$ Such tunability could endow organic cages that would incorporate rylene diimide units with a range of optoelectronic properties.

Therefore, we have explored the possibility to integrate them into organic cages. ${ }^{[18]}$ The design and synthesis of the chiral rylene cages $\mathbf{5}$ is shown in Scheme 1 and involves a $[3+2]$ condensation of a chiral rylene diamine with benzene-1,3,5-tricarbaldehyde. The rylene diimine cages 5 can be formed in high chemical yields and we developed an HPLC protocol to achieve samples of very high purity necessary for photochemical experiments (see below). The formation of the cages is highly stereoselective because only homochiral cages are observed when their synthesis starts with a racemic mixture of the diamines. This is in accord with DFT calculations that show that the energies of heterochiral cages incorporating both enantiomers of the diamines are very high. 


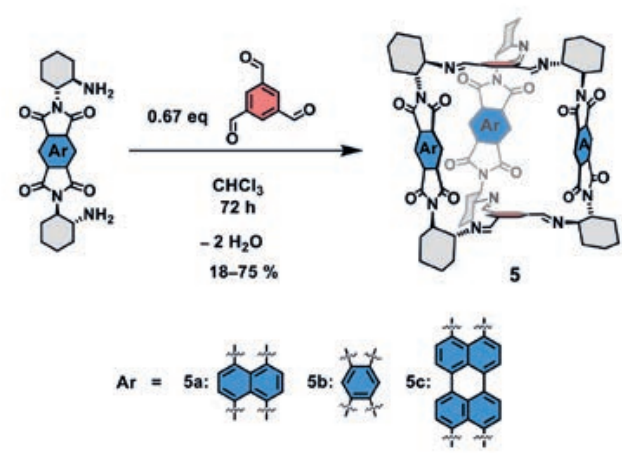

Scheme 1. The synthesis of POCs incorporating rylene diimides $\mathbf{5 a - c .}$

We determined the crystal structures of 5 by single crystal $\mathrm{X}$-ray diffraction analysis (Fig. 3). We discovered that the packing of the cages can be controlled by the nature of the crystallization solvent or by co-crystallization of cages with different chirality. When the cages are crystallized from electron-rich toluene, solvent molecules in the cage void create donor-acceptor pairs with individual rylene diimide units and are highly ordered. Thus, toluene fills the inner void of the cage and prevents the rylene diimide units from edge-to-face packing that requires partial penetration of a rylene diimide unit into the void of a neighboring cage. However, electron-poor nitrobenzene, a solvent with a similar size as toluene, allows the edge-to-face packing creating herringbone patterns transforming the crystal structure to a higher symmetry. Consequently, internal voids of the cages are interconnected and form two types of one-dimensional channels in the crystal (Fig. 3 ). In addition, PDI cage 5c benefits from additional PDI-PDI interactions because it has a higher aspect ratio than $\mathbf{5 a}$ or $\mathbf{5 b}$. As a result, new hexagonal extrinsic voids open in the crystal structure. Nitrobenzene has, however, a high boiling point and we could not successfully desolvate the crystals. Crystallization of the cages from THF, a low-boiling solvent that is also not expected to show any specific solvent-rylene diimide interactions, provided samples that, upon activation by dynamic vacuum or with supercritical $\mathrm{CO}_{2}$, showed permanent porosity and type I adsorption isotherm for $\mathbf{5} \mathbf{a}\left(S A_{\mathrm{BET}}=522 \mathrm{~m}^{2} \mathrm{~g}^{-1}\right)$. Cage $\mathbf{5 b}$ with three built-in pyromellitic diimides (PMDIs), on the other hand, showed initially a negligible uptake of $\mathrm{N}_{2}$ at $77 \mathrm{~K}$. However, as the partial pressure of $\mathrm{N}_{2}$ increased, a fast uptake of $\mathrm{N}_{2}$ suddenly occurred. We observed a non-closed desorption isotherm, which points to a gate-opening phenomenon related to kinetic gas trapping in $\mathbf{5 b}$. Thus, at low pressures, the empty channels in the activated material are blocked and a sudden structural rearrangement opening the voids takes place by increasing the external pressure. Such behavior is generally observed for flexible porous cages or soft porous crystals. ${ }^{[19]}$ POCs $\mathbf{5}$ showed a very promising selectivity and capacity of $\mathrm{CO}_{2}$ uptake compared to $\mathrm{N}_{2}$. Note that no specific nitrogen functionality is present in the structures of $\mathbf{5}$ but it can be easily installed by modifying either the rylene unit or the bridge.

Rylene diimides are useful chromophores, ${ }^{[17]}$ which motivated us to explore the photochemical behavior of $\mathbf{5} \mathbf{a}^{[18 a, 20]}$ and $\mathbf{5} \mathbf{c}^{[18 b]}$ in solution. For example, NDI is a very powerful oxidant in its excited state with a rapid intersystem crossing, ${ }^{[21]}$ while PDI with a lower excited state energy is, on the other hand, a well-known fluorophore with high fluorescence quantum yield. In cage 5a, an oxidation of the trisimine bridge by the excited NDI unit takes place creating singlet and triplet charge-separated (CS) states (Fig. 4, left). Thus, the electron transfer follows the rapid equilibration of the NDI singlet and triplet excited states known to the NDI chromophore. [21a] The singlet ${ }^{1} \mathrm{CS}$ state decays rapidly back to the ground state, while the triplet ${ }^{3} \mathrm{CS}$ state displays a lifetime longer than nanoseconds. Solvent polarity controls the yield of

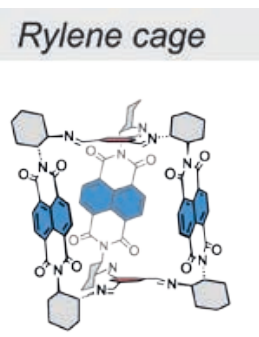

\section{$X R D$, sideview} $X R D$, topview

$5 a$
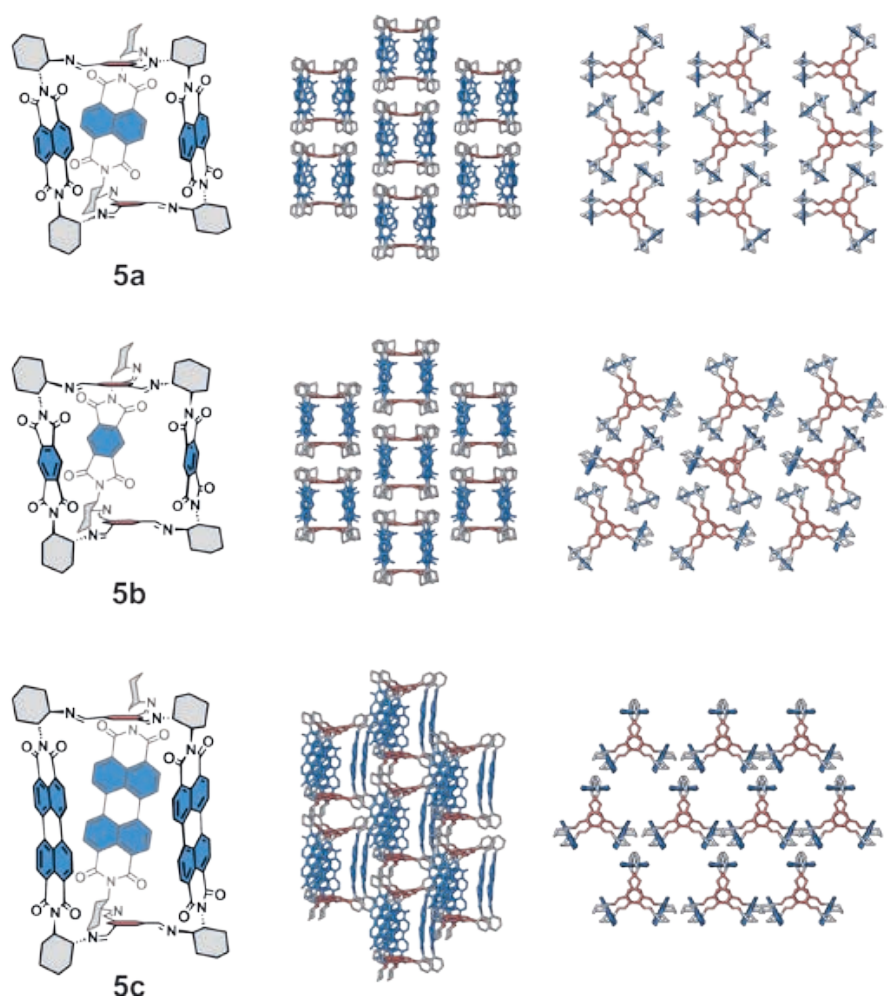

Fig. 3. (left) POCs incorporating rylene diimides, (middle) the sideview and (right) the topview of their solid-state superstructure determined by single crystal XRD. Hydrogen atoms and nitrobenzene solvent molecules are omitted for clarity. Figure adapted from ref. [18b].

the ${ }^{3} \mathrm{CS}$ state in $\mathbf{5 a}$ and it is markedly higher than what is found for analogous NDI monomers in the same solvents. ${ }^{[20]}$ We discovered that excitation of $\mathbf{5 c}$ in $\mathrm{CH}_{2} \mathrm{Cl}_{2}$ or benzonitrile leads to an efficient delayed fluorescence (Fig. 4, right) that we investigated by time-correlated single-photon counting and fluorescence quantum yields measurements. The delayed fluorescence does not originate from a reversible formation of an intracage PDI-PDI excimer in $\mathbf{5 c}$ because the absorption and emission spectra of $\mathbf{5 c}$ and a reference PDI monomer are nearly identical in all studied solvents. We observed identical behavior at different concentrations, which ruled out an involvement of any bimolecular processes. Finally, transient absorption spectroscopy showed that the delayed fluorescence is a consequence of a reversible intracage charge-separation event. Here, one PDI unit in $\mathbf{5 c}$ is reduced, while a second is oxidized. The formation of such CS state is feasible according to our electrochemical data, although our kinetic analysis revealed that the process must be considerably less exergonic than the PDI redox potentials and excited state energy indicate. The excited state dynamics in both cages thus suggest that the excited states in rylene diimide cages likely experience a different solvation than single rylene diimide chromophores. We assume that the aromatic surface of a rylene diimide exposed to the cage interior experiences a reduced adjustment of the solvent reaction field on the timescale of the observed photoexcited processes. This can be a consequence of a restricted rotation of the solvent guests in the cage cavities. Additional experiments and molecular simulations are necessary to support or disprove such an hypothesis. Nevertheless, our results demonstrate that CS states with sufficiently long lifetimes can be achieved in cages $\mathbf{5}$, which shall be tested in photoredox catalytic processes. Such work is in progress in our laboratory. 
Fig. 4. Excited state dynamics of $5 \mathbf{a}$ (left) and $\mathbf{5 c}$ (right). CS is a charge-separated state. Solid arrows indicate observed processes and dashed arrows indicate not observed processes. Numbers (in \%) denote the corresponding quantum yields in benzonitrile. Arrow that corresponds to the excitation in $\mathbf{5 c}$ (right) is omitted for clarity.

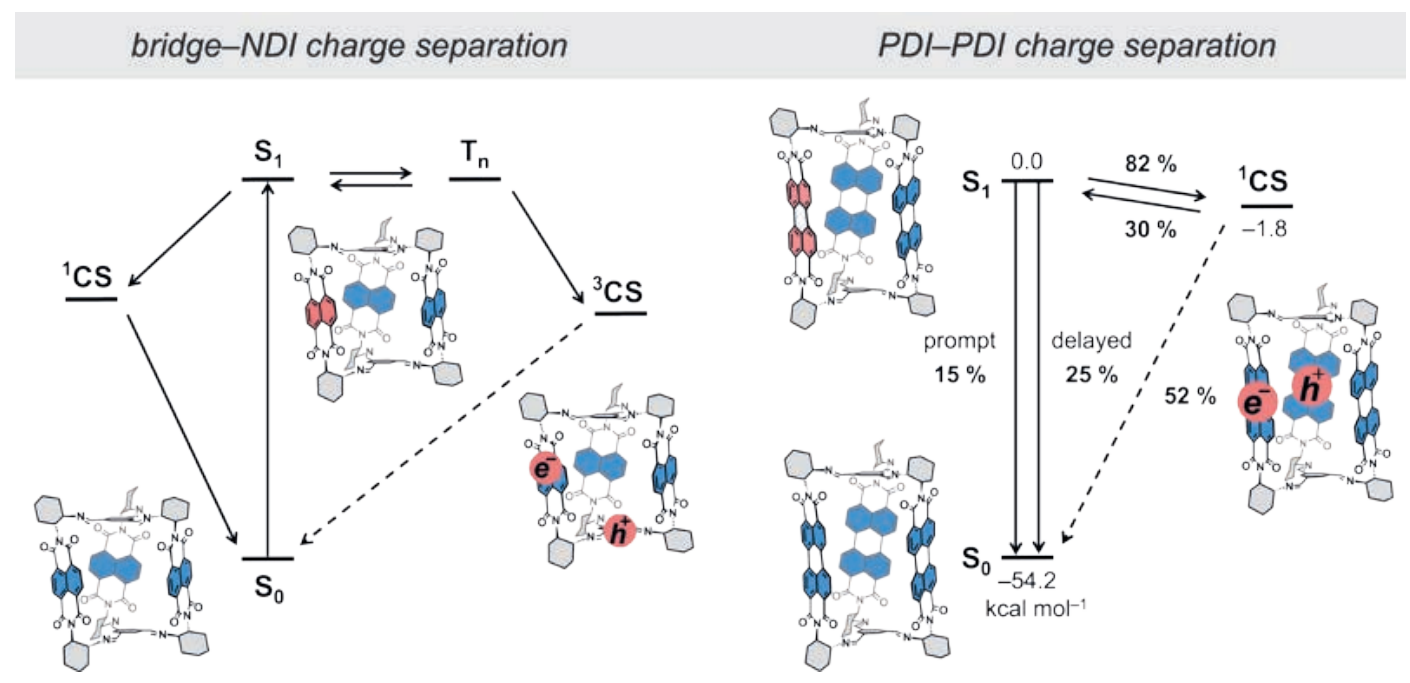

\section{Conclusion}

We presented the synthesis of porous organic cages via dynamic covalent chemistry and the impact the structures of individual cages may have on their porosity. We discussed the selectivity of porous organic cages to adsorb different gases and their ability to separate aromatic feedstock molecules, which highlights the great potential of porous organic cages with a variety of possible architectures in applications that require mass transport. In addition, we reviewed the recent development of porous organic cages that integrate an additional functionality, namely, photoactive molecules and we showed that these can lead to interesting photophysical properties and new applications, such as photocatalysis.

\section{Conflict of Interest}

The authors declare no competing financial interest.

\section{Acknowledgements}

We thank Prof. Marcel Mayor for generously hosting our group at the University of Basel and for his invaluable support of our research. We gratefully acknowledge the Department of Chemistry at the University of Basel and the Swiss National Science Foundation (SNSF, PZ00P2_174175) for their financial support. We thank Juraj Malinčík and Laurent Jucker for their invaluable help in proof-reading the manuscript. We also thank our Swiss collaborators, namely Prof. Michal Juríček (UZH), Prof. Ali Coskun (UniFr), and Prof. Eric Vauthey (UniGe) for wonderful scientific discussions that help drive our research forward. We wish to express our sincere gratitude to SCS and Metrohm for their generous support of the Best Oral Presentation Award.

Received: February 17, 2021

[1] O. M. Yaghi, J. Am. Chem. Soc. 2016, 138, 15507, https://doi.org/10.1021/jacs.6b11821

[2] a) O. M. Yaghi, H. Li, J. Am. Chem. Soc. 1995, 117, 10401, https://doi.org/10.1021/ja00146a033; b) J. Liu, J. Huang, L. Zhang, J. Lei, Chem. Soc. Rev. 2021, 50, 1188, https://doi.org/10.1039/D0CS00178C.

[3] a) A. P. Côté, A. I. Benin, N. W. Ockwig, M. O'Keeffe, A. J. Matzger, O. M. Yaghi, Science 2005, 310, 1166, https://doi.org/10.1126/science.1120411;b)K.Geng,T.He,R.Liu,S.Dalapati, K. T. Tan, Z. Li, S. Tao, Y. Gong, Q. Jiang, D. Jiang, Chem. Rev. 2020, 120, 8814, https://doi.org/10.1021/acs.chemrev.9b00550; c) H. M. El-Kaderi, J. R. Hunt, J. L. Mendoza-Cortés, A. P. Côté, R. E. Taylor, M. O’Keeffe, O. M. Yaghi, Science 2007, 316, 268, https://doi.org/10.1126/science.1139915.

[4] a) J. W. Colson, A. R. Woll, A. Mukherjee, M. P. Levendorf, E. L. Spitler, V. B. Shields, M. G. Spencer, J. Park, W. R. Dichtel, Science 2011, 332, 228, https://doi.org/10.1126/science.1202747; b) C. S. Diercks, O. M. Yaghi, Science 2017, 355, eaal1585, https://doi.org/10.1126/science.aal1585; c) Y Zhao, L. Guo, F. Gándara, Y. Ma, Z. Liu, C. Zhu, H. Lyu, C. A. Trickett, E.
A. Kapustin, O. Terasaki, O. M. Yaghi, J. Am. Chem. Soc. 2017, 139, 13166, https://doi.org/10.1021/jacs.7b07457.

[5] T. Hasell, A. I. Cooper, Nat. Rev. Mater. 2016, 1, 1, https://doi.org/10.1038/natrevmats.2016.53

[6] a) S. J. Rowan, S. J. Cantrill, G. R. L. Cousins, J. K. M. Sanders, J. F. Stoddart, Angew. Chem. Int. Ed. 2002, 41, 898, https://doi. org/10.1002/1521-3773(20020315)41:6<898::AID-ANIE898>3.0.CO;2E; b) M. E. Belowich, J. F. Stoddart, Chem. Soc. Rev. 2012, 41, 2003, https://doi.org/10.1039/C2CS15305J.

[7] T. Tozawa, J. T. A. Jones, S. I. Swamy, S. Jiang, D. J. Adams, S. Shakespeare, R. Clowes, D. Bradshaw, T. Hasell, S. Y. Chong, C. Tang, S. Thompson, J. Parker, A. Trewin, J. Bacsa, A. M. Z. Slawin, A. Steiner, A. I. Cooper, Nat. Mater. 2009, 8, 973, https://doi.org/10.1038/nmat2545.

[8] T. Mitra, K. E. Jelfs, M. Schmidtmann, A. Ahmed, S. Y. Chong, D. J. Adams, A. I. Cooper, Nat. Chem. 2013, 5, 276, https://doi.org/10.1038/nchem.1550.

[9] a) L. Chen, P. S. Reiss, S. Y. Chong, D. Holden, K. E. Jelfs, T. Hasell, M. A. Little, A. Kewley, M. E. Briggs, A. Stephenson, K. M. Thomas, J. A. Armstrong, J. Bell, J. Busto, R. Noel, J. Liu, D. M Strachan, P. K. Thallapally, A. I. Cooper, Nat. Mater. 2014, 13, 954, https://doi.org/10.1038/nmat4035; b) A. Kewley, A. Stephenson, L. Chen, M. E. Briggs, T. Hasell, A. I. Cooper, Chem. Mater. 2015, 27, 3207, https://doi.org/10.1021/acs.chemmater.5b01112; c) J. H. Zhang, S. M. Xie, L. Chen, B. J. Wang, P. G. He, L. M. Yuan, Anal. Chem. 2015, 87 7817, https://doi.org/10.1021/acs.analchem.5b01512; d) J. H. Zhang, S. M. Xie, B. J. Wang, P. G. He, L. M. Yuan, J. Chromatogr. A 2015, 1426, 174 https://doi.org/10.1016/j.chroma.2015.11.038.

[10] M. Souto, A. Santiago-Portillo, M. Palomino, I. J. Vitórica-Yrezábal, B. J. C. Vieira, J. C. Waerenborgh, S. Valencia, S. Navalón, F. Rey, H. García, G. Mínguez Espallargas, Chem. Sci. 2018, 9, 2413, https://doi.org/10.1039/C7SC04829G.

[11] S. Hiroto, Y. Miyake, H. Shinokubo, Chem. Rev. 2017, 117, 2910, https://doi.org/10.1021/acs.chemrev.6b00427.

[12] S. Hong, M. R. Rohman, J. Jia, Y. Kim, D. Moon, Y. Kim, Y. H. Ko, E. Lee, K. Kim, Angew. Chem. Int. Ed. 2015, 54, 13241, https://doi.org/10.1002/anie.201505531.

[13] a) Y. Jin, B. A. Voss, R. D. Noble, W. Zhang, Angew. Chem. Int. Ed. 2010 49, 6348, https://doi.org/10.1002/anie.201001517; b) Y. Jin, B. A. Voss, A Jin, H. Long, R. D. Noble, W. Zhang, J. Am. Chem. Soc. 2011, 133, 6650, https://doi.org/10.1021/ja110846c.

[14] a) X. Yu, B. Wang, Y. Kim, J. Park, S. Ghosh, B. Dhara, R. D. Mukhopadhyay, J. Koo, I. Kim, S. Kim, I. C. Hwang, S. Seki, D. M. Guldi, M. H. Baik, K. Kim, J. Am. Chem. Soc. 2020, 142, 12596, https://doi.org/10.1021/jacs.0c05339; b) R. D. Mukhopadhyay, Y. Kim, J. Koo, K. Kim, Acc. Chem. Res. 2018, 51, 2730 https://doi.org/10.1021/acs.accounts.8b00302.

[15] J. Koo, I. Kim, Y. Kim, D. Cho, I. C. Hwang, R. D. Mukhopadhyay, H. Song, Y. H. Ko, A. Dhamija, H. Lee, W. Hwang, S. Kim, M. H. Baik, K. Kim, Chem 2020, 6, 3374, https://doi.org/10.1016/j.chempr.2020.10.002.

[16] C. Liu, K. Liu, C. Wang, H. Liu, H. Wang, H. Su, X. Li, B. Chen, J. Jiang, Nat. Commun. 2020, 11, 1047, https://doi.org/10.1038/s41467-020-14831-x.

[17] a) M. Al Kobaisi, S. V. Bhosale, K. Latham, A. M. Raynor, S. V. Bhosale, Chem. Rev. 2016, 116, 11685, https://doi.org/10.1021/acs.chemrev.6b00160; b) N. Sakai, J. Mareda, E. Vauthey, S. Matile, Chem. Commun. 2010, 46, 4225, https://doi.org/10.1039/C0CC00078G; c) S. L. Suraru, F. Würthner, Angew. Chem. Int. Ed. 2014, 53, 7428, https://doi.org/10.1002/ anie.201309746; d) F. Würthner, C. R. Saha-Möller, B. Fimmel, 
S. Ogi, P. Leowanawat, D. Schmidt, Chem. Rev. 2016, 116, 962, https://doi.org/10.1021/acs.chemrev.5b00188; e) L. Chen, C. Li, K. Müllen, J. Mater. Chem. C 2014, 2, 1938, https://doi.org/10.1039/C3TC32315C.

[18] a) T. Šolomek, N. E. Powers-Riggs, Y. L. Wu, R. M. Young, M. D. Krzyaniak, N. E. Horwitz, M. R. Wasielewski, J. Am. Chem. Soc. 2017, 139, 3348, https://doi.org/10.1021/jacs.7b00233; b) H.-H. Huang, K. S. Song, A. Prescimone, A. Aster, G. Cohen, R. Mannancherry, E. Vauthey, A. Coskun, T. Šolomek, Chem. Sci. 2021, https://doi.org/10.1039/DISC00347J.

[19] a) S. Horike, S. Shimomura, S. Kitagawa, Nat. Chem. 2009, 1, 695, https://doi.org/10.1038/nchem.444; b) T. Mitra, X. Wu, R. Clowes, J. T. A. Jones, K. E. Jelfs, D. J. Adams, A. Trewin, J. Bacsa, A. Steiner, A. I. Cooper, Chem. Eur. J. 2011, 17, 10235, https://doi.org/10.1002/chem.201101631; c) Z. Wang, N. Sikdar, S. Q. Wang, X. Li, M. Yu, X. H. Bu, Z. Chang, X. Zou, Y. Chen, P. Cheng, K. Yu, M. J. Zaworotko, Z. Zhang, J. Am. Chem. Soc. 2019, 141, 9408, https://doi.org/10.1021/jacs.9b04319.

[20] A. Aster, C. Rumble, A.-B. Bornhof, H.-H. Huang, N. Sakai, T. Šolomek, S. Matile, E. Vauthey, Chem. Sci. 2021, https://doi.org/10.1039/DISC00285F.
[21] a) O. Yushchenko, G. Licari, S. Mosquera-Vazquez, N. Sakai, S. Matile, E. Vauthey, J. Phys. Chem. Lett. 2015, 6, 2096, https://doi.org/10.1021/acs.jpclett.5b00882; b) P. Ganesan, J. Baggerman, H. Zhang, E. J. R. Sudhölter, H. Zuilhof, J. Phys. Chem. A 2007, 111, 6151, https://doi.org/10.1021/jp0719558.

\section{License and Terms}

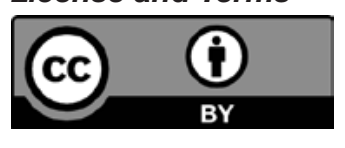

This is an Open Access article under the terms of the Creative Commons Attribution License CC BY 4.0. The material may not be used for commercial purposes.

The license is subject to the CHIMIA terms and conditions: (http:// chimia.ch/component/sppagebuilder/?view=page \&id=12).

The definitive version of this article is the electronic one that can be found at https://doi.org/10.2533/chimia.2021.285 